\title{
Hemiatrophy Syndrome with Secondary Respiratory Failure Cor- rected with Noninvasive Ventilation
}

\section{Lorena Picasso Simón and Salvador Díaz Lobato*}

\author{
Department of Pneumology, Ramón y Cajal Teaching Hospital, Madrid, Spain
}

*Corresponding author: Salvador Díaz Lobato, Department of Pneumology, Ramon y Cajal Teaching Hospital, Carretera de Colmenar Viejo, Km. 9,100, 28034 Madrid, Spain, Tel: +003-4913-368-133, E-Mail: sdiazlobato@gmail.com

\begin{abstract}
Hemiatrophy syndrome is a rare disease defined by the occurrence of a body hemiatrophy.

We describe the case of a 79-year-old man who presented respiratory failure secondary to left body hemiatrophy which was presented since early childhood. Clinical and images findings support the diagnosis of hemiatrophy syndrome complicated with hypercapnic respiratory insufficiency secondary to a restrictive ventilatory disorder.

This case report further characterizes this rare syndrome which can be effectively treated with noninvasive ventilation in cases where a hyercapnic respiratory failure appears.
\end{abstract}

\section{Keywords}

Hemiatrophy syndrome, Respiratory failure, Noninvasive ventilation

\section{Introduction}

Body hemiatrophy (HA) is a rare syndrome. The clinical hallmarks include the occurrence of atrophy on one side of the body which is often present but not necessarily noted by patient since early childhood [1,2]. It is characterized by asymmetry of size of the extremities on one side of the body, being shorter than the contralateral one. However, the abnormalities not only focus on extremities, but also can affect the lung and the brain. In some patients it has been described secondarily the appearance of respiratory insufficiency and neurological disorders like Parkinsonism, epilepsy and others. We present a patient with HA suffering of hypercapnic respiratory failure secondary to the ventilatory restrictive alteration that was effectively treated with noninvasive mechanical ventilation (NIV). In our knowledge, this is the first report in the literature of a patient with respiratory failure due to HA treat successfully with NIV.

\section{Case Report}

A 79-year-old man was referred to the pulmonology department complaining of progressive dyspnea on minimal efforts. The patient had been diagnosed of hypertension, dyslipemia, chronic ischemic cardiopathy with old myocardial infarction at lower surface, colon diverticulosis with polyps and internal hemorrhoids. He was moderate smoker (15 pack-years). He did not refer any allergies. The physical examination revealed tachypnea ( 25 breaths per minute), an oxygen saturation by pulse-oximetry $\left(\mathrm{SpO}_{2}\right) 76 \%$, and a decrease level of consciousness (Glasgow scale 13/15). The pulmonary auscultation showed decreased breath sounds at the left lung. An arterial gasometry showed pH: 7.48; partial pressures of carbon dioxide $\left[\mathrm{pCO}_{2}\right] 46 \mathrm{mmHg}$; partial pressures of arterial oxygen $\left[\mathrm{pO}_{2}\right] 58 \mathrm{mmHg}$; bicarbonate concentration $\left[\mathrm{HCO}_{3}\right] 34.3 \mathrm{mmol} /$ /iter. In addition, he had mild hemifacial asymmetry and weakness, with left ptosis. Left pectoral muscle, left arm and leg atrophy were significantly smaller (Figure 1). Computerized tomography showed a decrease in volume of the left lung being evident the pectoral atrophy (Figure 2). A spirometry test revealed a moderate restrictive ventilatory pattern: FVC $1.70 \mathrm{~L}$ (54.7\%), FEV1 1.26 L (54.2\%), FEV1/FVC $73.83 \%$. Interestingly, in renal and urinary ecography there were no alterations being normal kidneys and bladder.

The patient was hospitalized 7 days and during the 


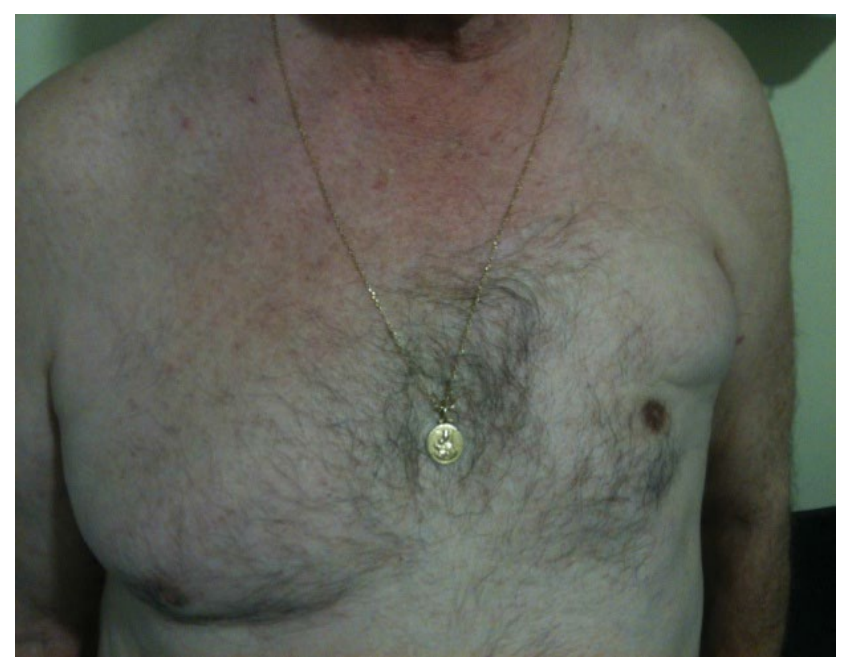

Figure 1: Left pectoral smaller than right pectoral.

hospitalization period was managed with antibiotics (amoxicillin clavulanic), steroids and normal diet. Noninvasive mechanical ventilation was established with bilevel pressure (Stellar ${ }^{\circledR} 100$, ResMed) with a nasal mask. Initial settings were inspiratory positive airway pressure (IPAP) of $16 \mathrm{~cm} \mathrm{H}_{2} \mathrm{O}$ and expiratory positive airway pressure (EPAP) of $6 \mathrm{~cm} \mathrm{H}_{2} \mathrm{O}$. NIV was well tolerated by the patient and domiciliary nocturnal ventilation was prescribed. Six-months later, the patient was asymptomatic and an arterial gasometry showed normal values, having disappeared the hypercapnic respiratory failure.

\section{Discussion}

The hemiatrophy syndrome is an uncommon disease that is not very well known and is hard to find articles related to this syndrome. Most of the articles talk about hemiparkinsonism-hemiatrophy syndrome or other neurological syndromes. Hemiparkinsonism-hemiatrophy syndrome is a relatively rare cause of secondary Parkinsonism [3] that usually begins in the third or fourth decade of life. It is characterized by unilateral body atrophy and ipsilateral Parkinsonism findings with slow progression [4]. With regard to hemiatrophy syndrome, is an unusual disease characterized by the decrease in the size of hemibody affecting: face, brain, extremities, thorax and lung. The physical changes have no more implications than the esthetic, although it can entail pshychological problems especially in adolescence but this has not

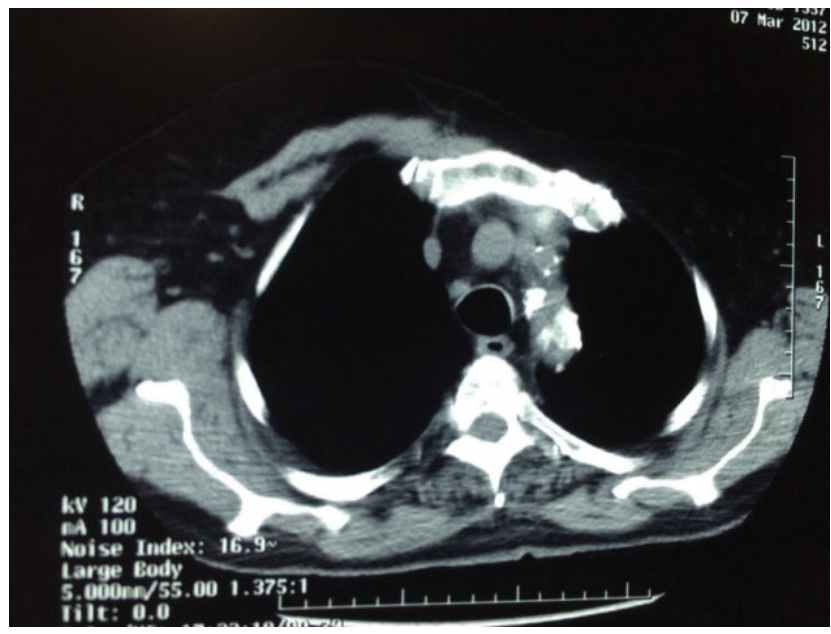

Figure 2: Absence of left pectoral muscle. Volume of left lung decreased.

been studied. In relation to the variations in lungs, the most important aspect is the decrease of the volume that entails a respiratory insufficiency. This is a restrictive thoracic disease that in our case was treated with noninvasive ventilation having a good response. NIV has been successfully used for a wide variety of restrictive thoracic diseases, including thoracic cage abnormalities (e.g. chest wall deformities, kyphoscoliosis, spinal cord injury, and sequelae of polio), in addition to both rapidly and slowly progressive neuromuscular conditions [5]. In our opinion HA has to be incorporated to the list of causes of respiratory failure where NIV should be taken into account.

\section{References}

1. Tessitore A, Russo A, Cirillo M, Giordano A, Marcuccio L, et al. (2010) Hemiparkinsonism and hemiatrophy syndrome: A rare observation. Clin Neurol Neurosurg 112: 524-526.

2. Wijemanne S, Jankovic $J$ (2007) Hemiparkinson-hemiatrophy syndrome. Neurology 69: 1585-1594.

3. Silvers DS, Menkes DL (2009) Hemibody mirror movements in hemiparkinsonism-hemiatrophy syndrome. J Neurol Sci 287: 260-263.

4. Ayromlou H, Najmi S, Arami MA (2011) Hemiparkinsonism-hemiatrophy syndrome. Arch Iran Med 14: 152-154.

5. Wahab R, Basner RC (2013) Nocturnal non-invasive ventilation for cardio-respiratory disorders in adults. Expert Rev Respir Med 7: 615-629. 